International Journal of Linguistics, Literature and Translation

ISSN: 2617-0299 (Online); ISSN: 2708-0099 (Print)

DOI: 10.32996/ijltt

Journal Homepage: www.al-kindipublisher.com/index.php/ijltt

\title{
Translating The Difference in The Land of An African Sultan by Walter B. Harris
}

\section{Dr. Soufiane Laachiri 8 (D)}

ترجمة الاختلاف في مملكة السلطان الإفريقي لوالتر بورتن هاريس

Researcher, Abdelmalik Essaadi University, Morocco

$\triangle$ Corresponding Author: Hakeem Salmon, E-mail: soufiane.laachiri@gmail.com

\section{ARTICLE INFORMATION}

Received: July 11, 2021

Accepted: August 07, 2021

Volume: 4

Issue: 8

DOI: $10.32996 /$ ijllt.2021.4.8.3

\section{KEYWORDS}

The Land of An African Sultan, Walter B. Harris

\section{ABSTRACT}

The present article focuses on the discursive translation of colonial knowledge as a set of complex statements of power and exclusion in Harris' The Land of An African Sultan. This discursive process of translation acts as a continuum for the main foundations upon which post colonial consciousness, as articulated by such third world critics as Edward Said, Homi Bhabha and Gayatri Spevak, is generically based.The book is also a scientific encyclopedia which highlights a historical era with all its political and military events. It also describes the Moroccan society, its people, its political regime, its Zawaya, its culture, its customs and beliefs, its climate and geography, its architecture and landscape, its races, and even its demons. In so doing,Harris was paving the way for European imperialism through his constant descriptions and representations of the Moroccan other.His travels throughout the entire country as a traveler, and his reports about that as a journalist of The Times have explained the close relationship between Colonialism and travel writing. This relationship, being mutually a sustained way to create images of vacant spaces over time and place, highlights settlement in, and improvement of 'primitive' lives which were viewed as 'uncivilized'. It also justifies the colonial enterprise which came to escalate development of the colonized land and work for the welfare of its peoples . In this dual process of interaction, translation plays significant roles.

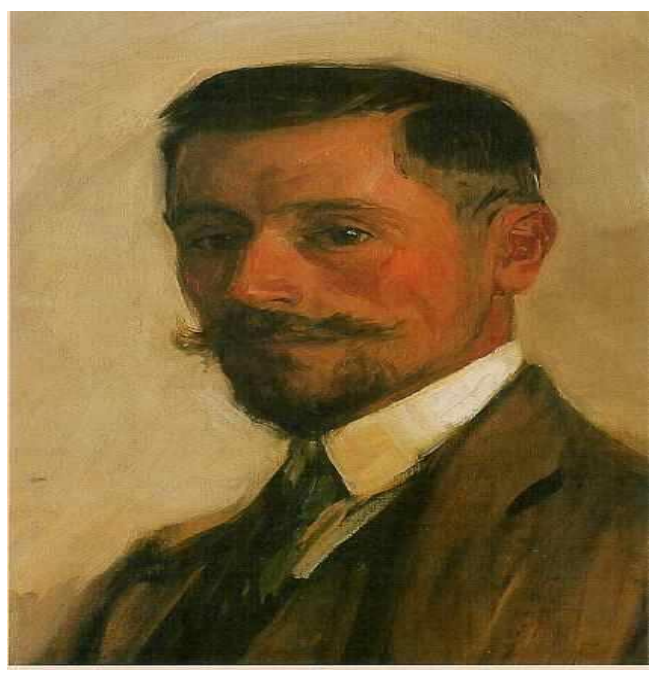

Walter B.Harris

(From the album of Younes Cheikh Ali) 


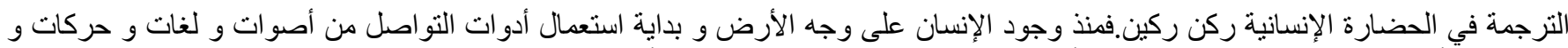

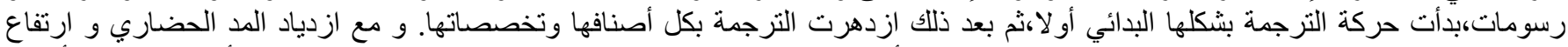

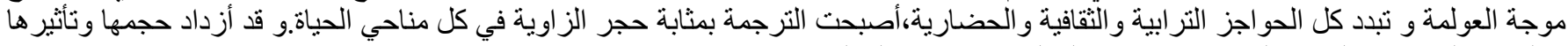

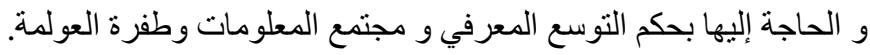

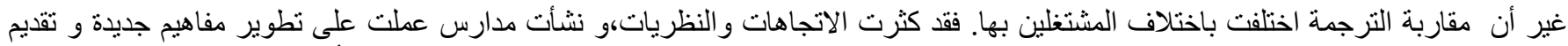

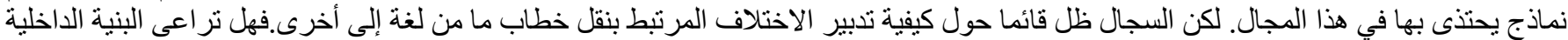

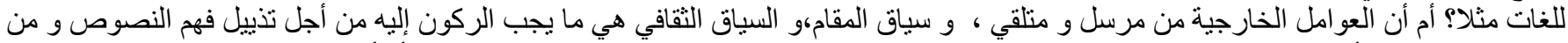

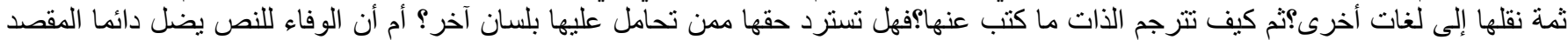

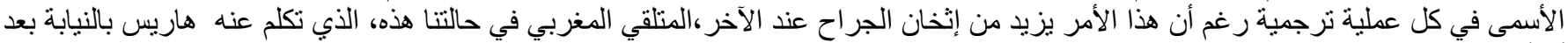

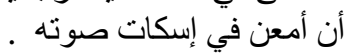

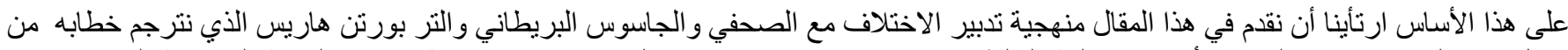

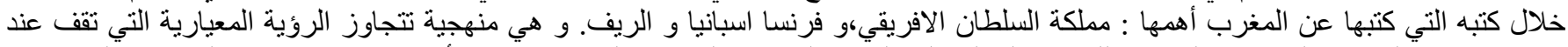

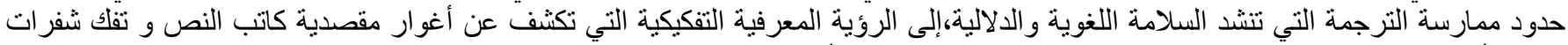

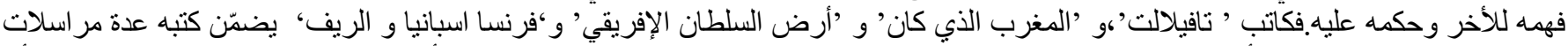

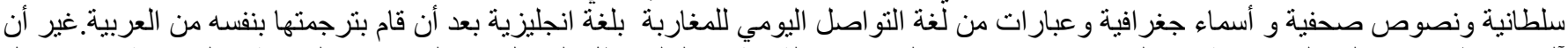

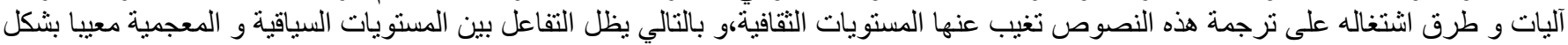

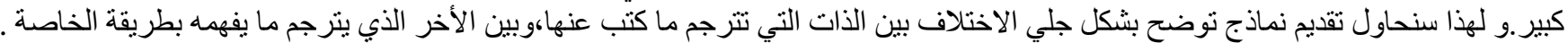

والتز هاريس في مواجهة الآخر:

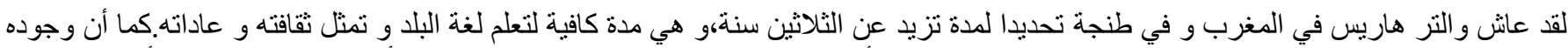

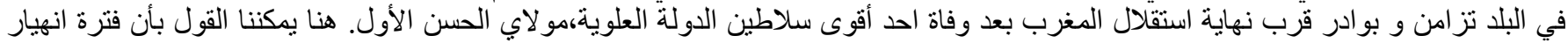

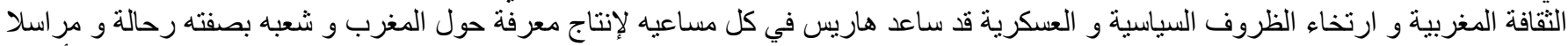

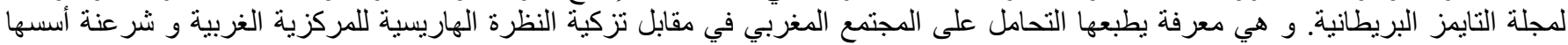

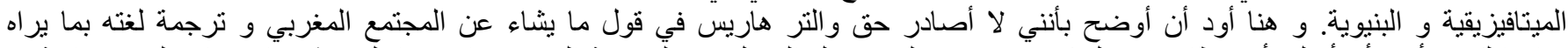

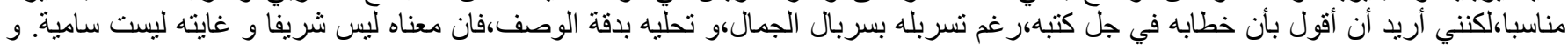

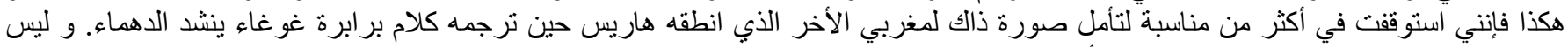

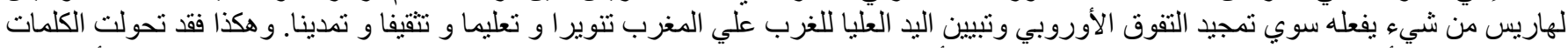

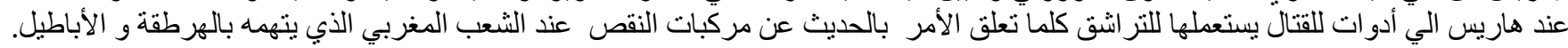

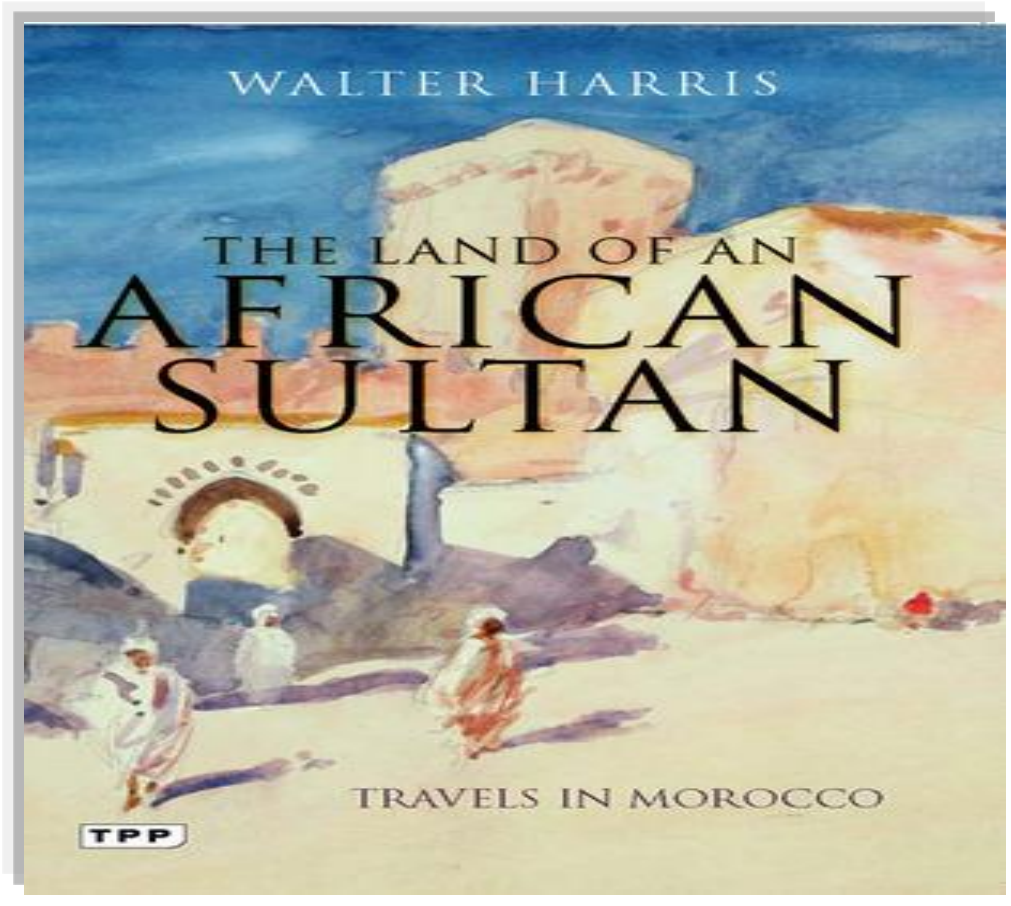

Www.googleimages.com 


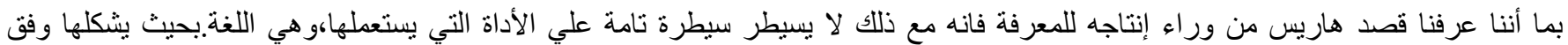

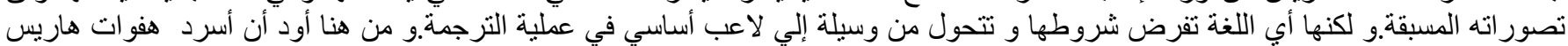

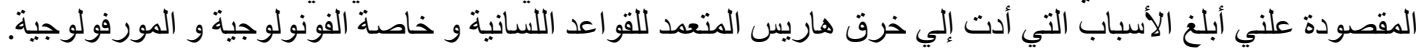

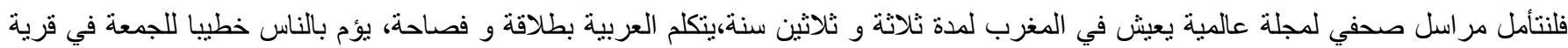

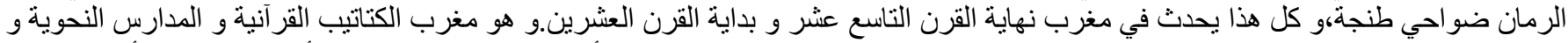

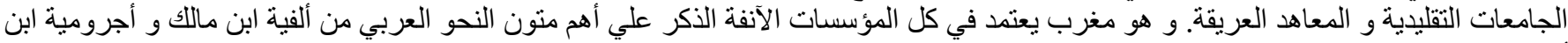

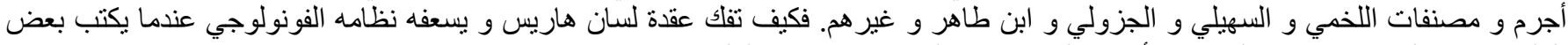
الكلمات بالانجليزية و يحافظ على نفس نأثير ها الصوني في العربية،ورو من هذه الكلمات:

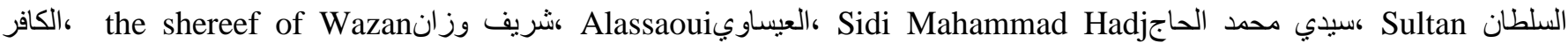

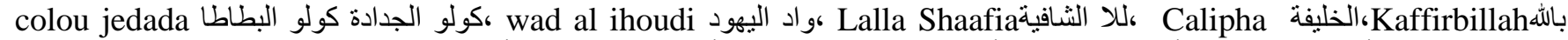
colou patata .

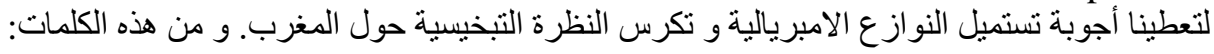

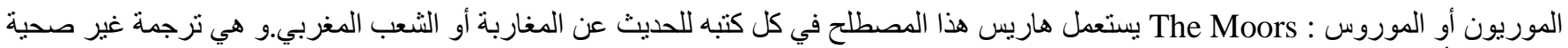

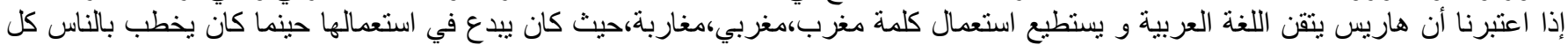

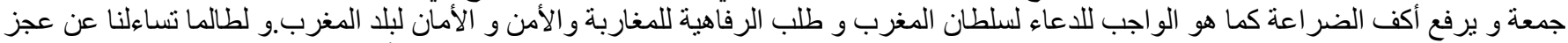

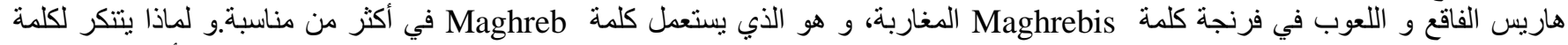
هoroccans الموجودة سلفا في كل القو اميس الانجليزية،و لماذا لا يقوب علي نسخ الكلمة العربية و تحوير هأ كما هو معمول به في أدبيات الترجمة؟

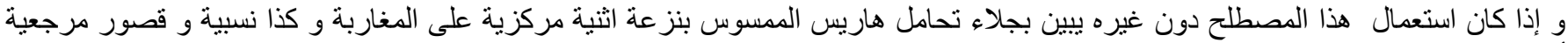

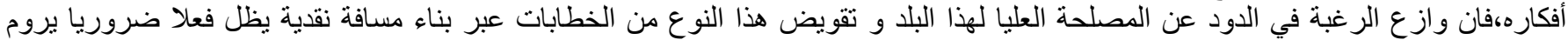

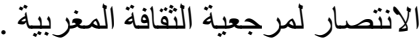

إن التعدي على حرمة اللغة عند هاريس ما هو إلا دليل على أن الأجندات السياسية والأيديولوجية تبيح منى ما تطلب الأمر ذلك الانفلات من قو انين الألسنية لكي لا ينقض كرمةتبنا عرى المركزية الغربية و أسسها الميتافيزيقية.

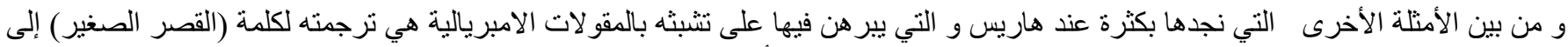

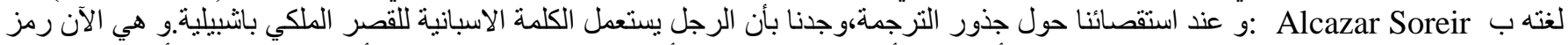

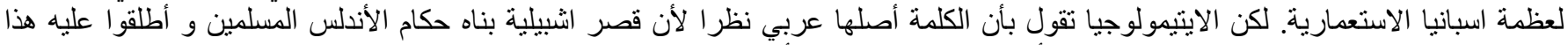

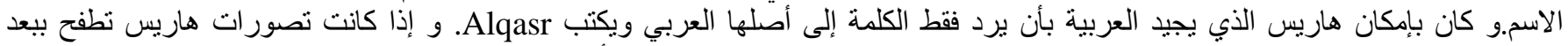

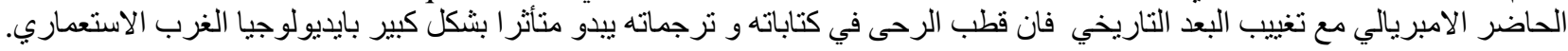

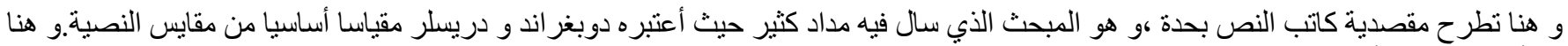

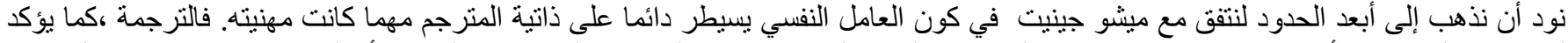

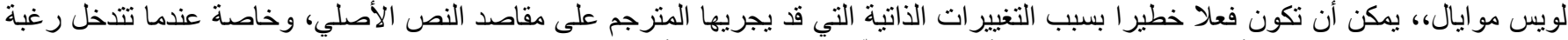
المترجم وشخصيته، عن وعي أو لاوعي، في ترجمنه، أو عندما يحكّم السياق السياسي أو الثقافي الذي ينتمي إلبه. و هذا بالضبط ما حدث لهاريس.

على سبيل الدحض و الاستتناج:

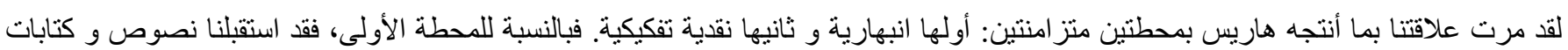

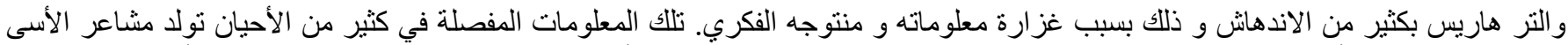

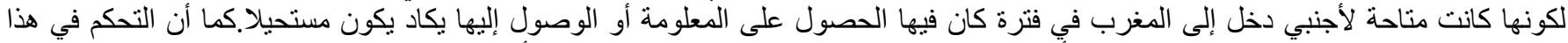

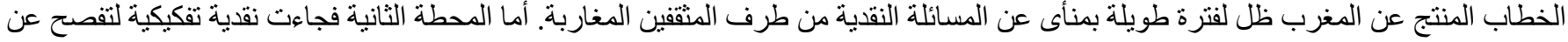
وعي ذاتي، وعي الذات الثقافية التي تحاول إعادة استملاك نفسهاو الإفصاح عن و عيهاو التخلص ممن احتكر الرواية عنها.

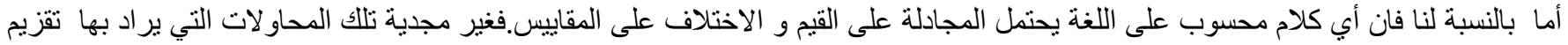

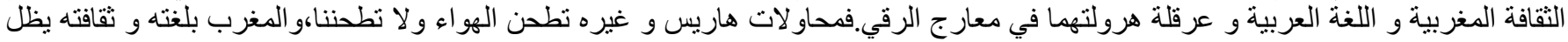


الدكتور سفيان العشيري، حاصل عل الإجازة في الأدب الانجليزي من كلية الآداب و العلوم الإنسانية بتطوان.كما حصل على شهادة الماجستير في

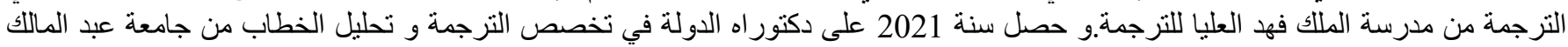

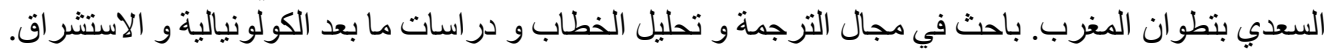

المصادر العربية:

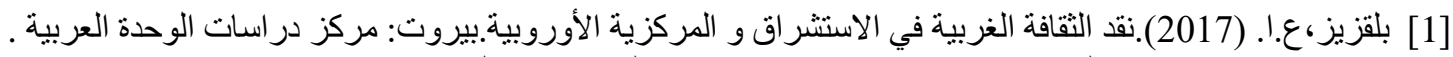

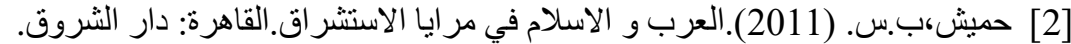

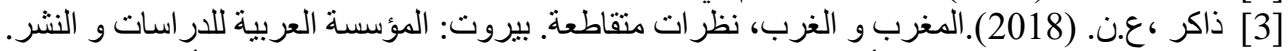

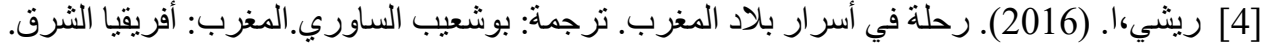

[1] Beaugrande, R. de \& Dressler. (1981). Introduction to textlinguistics.London : Longman.

المصادر الأجنبية : n n

[2] Mehrach,M. (1997). Towards a text-based model for translation evaluation.Ridderkerk :Ridderprint.

[3] Said, E. (1993). Culture and imperialism.Great Britain :Vintage.

[4] Said, E. (1978). Orientalism. UK :Routledge \& Kegan Paul Ltd.

[5] Said.E. (1993). Culture and Imperialism. Great Britain :Vintage. 